\title{
Symmetry-Protected Scattering in Non-Hermitian Linear Systems
}

\author{
L. Jin ${ }^{1, *}$ and Z. Song ${ }^{1}$ \\ ${ }^{1}$ School of Physics, Nankai University, Tianjin 3000\%1, China
}

\begin{abstract}
Symmetry plays fundamental role in physics and the nature of symmetry changes in non-Hermitian physics. Here the symmetry-protected scattering in non-Hermitian linear systems is investigated by employing the discrete symmetries that classify the random matrices. The even-parity symmetries impose strict constraints on the scattering coefficients: the time-reversal $(C$ and $K)$ symmetries protect the symmetric transmission or reflection; the pseudo-Hermiticity ( $Q$ symmetry) or the inversion $(P)$ symmetry protects the symmetric transmission and reflection. For the inversion-combined time-reversal symmetries, the symmetric features on the transmission and reflection interchange. The odd-parity symmetries including the particle-hole symmetry, chiral symmetry, and sublattice symmetry cannot ensure the scattering to be symmetric. These guiding principles are valid for both Hermitian and non-Hermitian linear systems. Our findings provide fundamental insights into symmetry and scattering ranging from condensed matter physics to quantum physics and optics.
\end{abstract}

Introduction. - Quantum transport and light scattering depend on the properties of media [1, 2]. In optics, the Lorentz reciprocity is fundamental due to the symmetric permittivity tensor; which results in symmetric transmission when the input and output channels are interchanged [2, 3]. Breaking reciprocity is important for the light flow molding and the nonreciprocity plays a crucial role in tailoring the light field. The optical isolator has a propagation direction dependent transmission [49]. The reciprocity breaks in the magneto-optical materials $10-14$ and the asymmetric nonlinear optical structures $[15-19]$. In comparison, the nonlinear systems are preferable for their integrability; however, in addition to the requirement of high intensity, the desirable light flow engineering is also comparably difficult. Alternatively, temporal modulation of the propagation constant in the linear waveguides realizes magnetic-free nonreciprocity through the synthesized magnetic flux [20 31], being advantageous for the scalable integrated devices in a wide range of optical, radio, and audible frequencies [3].

Recently, reciprocal and nonreciprocal anomalous scattering are demonstrated in non-Hermitian systems [32]. The scattering dynamics closely relates to the symmetries of the scattering center. The reciprocity still holds in the parity-time-symmetric non-Hermitian metamaterials that judiciously incorporate gain and loss 33 35]. The inversion symmetry guarantees the symmetric transmission and reflection 36 43]; the time-reversal symmetry ensures the symmetric reflection [44, 45]; and the paritytime symmetry protects the symmetric transmission $45-$ 95]. Nevertheless, these conclusions are insufficient to fully capture the symmetric properties of scattering and the role played by the symmetry for an arbitrary linear system [96]. More important, the nature of symmetry changes in non-Hermitian physics [97, 98]. Now, the fundamental principles for the symmetry-protected scattering remain concealed and are urgent to be settled as the rapid progresses in non-Hermitian physics 99 106.

In this Letter, we report the symmetry-protected scattering in non-Hermitian linear systems and reveal the fundamental roles played by the symmetries. We show that the internal symmetries $C, K, Q, P$ that classify the non-Hermitian random matrices protect the symmetric transmission and/or reflection 107]. The nonHermiticity helps breaking the symmetry protection and enables a various of intriguing asymmetric scattering in the linear photonic lattices, which has promising applications as optical diode, isolator, and modulator. The scattering theory tackles problems including light propagation in dissipative metamaterial, on-chip functional photonic device design, and quantum transport manipulation in mesoscopic.

Symmetries. - The non-Hermitian scattering center $H_{c}$ is classified under the discrete symmetries 107, 108]

$$
\begin{aligned}
& C \text { sym. : } H_{c}=\epsilon_{c} c H_{c}^{T} c^{-1}, c c^{*}= \pm \mathbf{1}, \\
& K \text { sym. : } H_{c}=\epsilon_{\mathbb{k}} \mathbb{k} H_{c}^{*} \mathbb{k}^{-1}, \mathbb{R} \mathbb{k}^{*}= \pm \mathbf{1}, \\
& Q \text { sym. : } H_{c}=\epsilon_{q} q H_{c}^{\dagger} q^{-1}, q^{2}=\mathbf{1}, \\
& P \text { sym. : } H_{c}=\epsilon_{p} p H_{c} p^{-1}, p^{2}=\mathbf{1} .
\end{aligned}
$$

$H_{c}^{T}, H_{c}^{*}$, and $H_{c}^{\dagger}$ are the transpose, complex conjugation, and Hermitian conjugation of $H_{c}$, respectively. $c, \mathbb{k}, q$, $p$ are unitary operators. The signs $\epsilon_{c, \mathbb{k}, q, p}= \pm 1$ denote the parity of symmetries $C, K, Q, P$. For non-Hermitian scattering center $\left(H_{c} \neq H_{c}^{\dagger}\right)$, both the $C$ and $K$ symmetries relate to the time-reversal symmetry $\epsilon_{c, \mathbb{k}}=+1$ and the particle-hole symmetry $\epsilon_{c, \mathbb{k}}=-1$ [98]. The $Q$ symmetry is pseudo-Hermitian for $\epsilon_{q}=+1$ and pseudoanti-Hermitian for $\epsilon_{q}=-1$ (also referred to as the chiral symmetry 98]). The $P$ symmetry with even-parity $\epsilon_{p}=+1$ is the inversion symmetry if $p$ is the identity matrix rotated by 90 degrees. The $P$ symmetry with the odd-parity $\epsilon_{p}=-1$ is the sublattice symmetry. The eight symmetries form an $E 8$ Abelian group [109].

The even parity $\left(\epsilon_{c, \mathbb{k}, q, p}=+1\right)$ symmetries, including time-reversal symmetry, pseudo-Hermiticity, and generalized inversion symmetry, can result in symmetric transmission and/or reflection; the constraints imposed by the symmetries $C, K$ are that either the transmission or the reflection is symmetric; both the symmetries $P, Q$ 

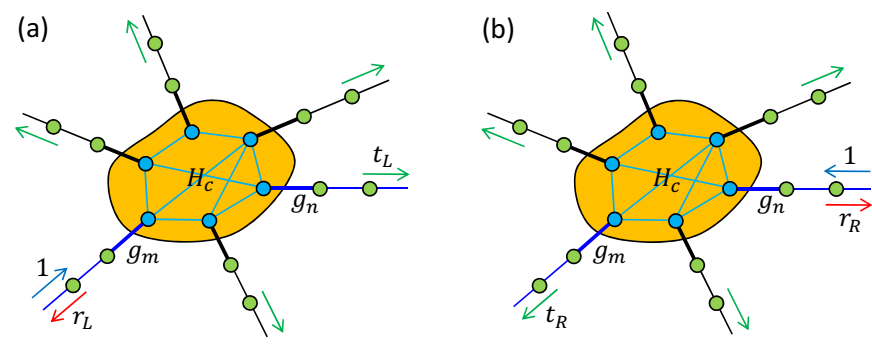

FIG. 1. Schematic of a multi-port discrete scattering system. The orange area indicates an $N$-site scattering center $H_{c}$. The connection coupling between the lead $j$ and the scattering center site $j$ is denoted as $g_{j}(j \in[1, N])$. (a) Forward incidence in the lead- $m$. (b) Backward incidence in the lead- $n$.

can induce symmetric transmission and reflection. The symmetry-protected transmission or reflection was observed in many experiments [37, 54, 55, 64, 71, 110 112]. In contrast, the odd-parity $\left(\epsilon_{c, \mathbb{k}, q, p}=-1\right)$ symmetries, including particle-hole symmetry, chiral symmetry, and sublattice symmetry, do not ensure the transmission or reflection to be symmetric because they cannot impose any symmetric constraint on the scattering coefficients.

Scattering formalism. - We consider a general multiport linear scattering center to elucidate the symmetry protection. In Fig. 1, the scattering center is a timeindependent $N$-site network (shaded in orange). The schematic models physical systems including the coupled resonators [8, 63, 113, 114], coupled waveguides 37, 51, 55, 71, 115], and optical lattices [62, 110, 116, 117]. The solid circles stand for the resonators, the waveguides, and the sites of the optical lattice. The solid lines represent the couplings. The leads are uniform lattice chains with the coupling strength $J$. For identical lead couplings, the scattering features are fully determined by the properties of the scattering center. The $j$-th lead is connected to the scattering center site $j$ at the coupling strength $g_{j}$. The arrows illustrate the scattering for the individual incidence in the $m$-th and $n$-th leads, respectively. The outgoing waves in red (green) are the reflections (transmissions). For more than one inputs, the scattering wavefunction is a superposition of wavefunctions of separately injecting each individual input; thus, the scattering properties are fully captured by the scattering of the individual input.

In the coupled mode theory 118 120], the equation of motion for the monochromatic light field amplitude $\phi_{l, j}^{k}(s)=\psi_{l, j}^{k}(s) e^{-i \omega t}$ in the $j$-th lead is

$$
i \dot{\phi}_{l, j}^{k}(s)=\omega_{0} \phi_{l, j}^{k}(s)+J \phi_{l, j}^{k}(s-1)+J \phi_{l, j}^{k}(s+1),
$$

for the site $|s| \geq 1[63$, 121]. The dispersion relation supported by the leads is $\omega=\omega_{0}+2 J \cos k$ for the incident momentum $k$ 8, 81], obtained from the steady-state solution of the light field amplitudes [122, 123]. The resonant incidence has frequency $\omega_{0}$. The equations of motion for the light field in the scattering center are

$$
i\left(\begin{array}{c}
\dot{\phi}_{c, 1}^{k} \\
\vdots \\
\dot{\phi}_{c, N}^{k}
\end{array}\right)=\left(\omega_{0} \mathbf{1}+H_{c}\right)\left(\begin{array}{c}
\phi_{c, 1}^{k} \\
\vdots \\
\phi_{c, N}^{k}
\end{array}\right)+\left(\begin{array}{c}
g_{1} \phi_{l, 1}^{k}(1) \\
\vdots \\
g_{N} \phi_{l, N}^{k}(1)
\end{array}\right),
$$

where the $N \times N$ matrix $H_{c}$ characterizes the scattering center and $\mathbf{1}$ is the $N \times N$ identity matrix. $\phi_{c, j}^{k}$ is the light field amplitude of the scattering center site $j . \phi_{l, j}^{k}(1)$ is the light field amplitude of the connection site on the lead $j . g_{j}$ is chosen $J$ or 0 without loss of generality to indicate the presence or absence of the lead $j$. For other $g_{j}$, the connection sites are counted as part of the scattering center and the connection couplings remain $J$. Setting $\phi_{c, j}^{k}=\psi_{c, j}^{k} e^{-i \omega t}$, we have $d \psi_{c, j}^{k} / d t=0$ at the steady-state; and the equations of motion reduce to

$$
\omega\left(\begin{array}{c}
\psi_{c, 1}^{k} \\
\vdots \\
\psi_{c, N}^{k}
\end{array}\right)=\left(\omega_{0} \mathbf{1}+H_{c}\right)\left(\begin{array}{c}
\psi_{c, 1}^{k} \\
\vdots \\
\psi_{c, N}^{k}
\end{array}\right)+\left(\begin{array}{c}
g_{1} \psi_{l, 1}^{k}(1) \\
\vdots \\
g_{N} \psi_{l, N}^{k}(1)
\end{array}\right) .
$$

In the multi-port scattering center, we consider the scattering properties of input and output in the leads $m$ and $n$. The steady-state equations of motion for the multi-port scattering system are equivalent to that for a two-port scattering system with leads $m$ and $n$. Each of the other lead $j(j \neq m, n)$ effectively reduces into an additional on-site self-energy term of the scattering center site $j$ in the equivalent scattering center $H_{c}^{\prime}$ [123]. Notably, the wavefunction in the additional lead $j(j \neq m, n)$ is outgoing wave $\psi_{l, j}^{k}(s)=t_{j} e^{i k s}$, and the wavefunction continuity yields $\psi_{l, j}^{k}(0)=\psi_{c, j}^{k}$. Thus, we have the relation $g_{j} \psi_{l, j}^{k}(1)=g_{j}^{2} J^{-1} e^{i k} \psi_{l, j}^{k}(0)=g_{j}^{2} J^{-1} e^{i k} \psi_{c, j}^{k}$; consequently, the second term $g_{j} \psi_{l, j}^{k}(1)$ in Eq. (7) results in an extra self-energy $g_{j}^{2} J^{-1} e^{i k}$ for the scattering center site $j$ in the equations of motion [124], and the multi-port scattering center is effectively characterized by the two-port scattering center $H_{c}^{\prime}=H_{c}+$ $J^{-1} e^{i k} \operatorname{diag}\left(\cdots, g_{m-1}^{2}, 0, g_{m+1}^{2}, \cdots, g_{n-1}^{2}, 0, g_{n+1}^{2}, \cdots\right)$ with additional on-site complex self-energies except for the scattering center sites $m$ and $n$. Therefore, the scattering properties of the multi-port scattering center $H_{c}$ are completely determined from analyzing the two-port scattering center $H_{c}^{\prime}$, and we focus on investigating the scattering properties of the two-port scattering center.

We take $g_{m}=g_{n}=J$ and $g_{j}=0(j \neq m, n)$. From Eq. (7), the wavefunctions for the scattering center sites $m$ and $n$ satisfy

$$
\begin{gathered}
\psi_{c, m}^{k}=-\Delta_{m m}^{-1} J \psi_{l, m}^{k}(-1)-\Delta_{m n}^{-1} J \psi_{l, n}^{k}(1), \\
\psi_{c, n}^{k}=-\Delta_{n m}^{-1} J \psi_{l, m}^{k}(-1)-\Delta_{n n}^{-1} J \psi_{l, n}^{k}(1),
\end{gathered}
$$


where $\Delta_{m n}^{-1}$ is the element of the $m$-th row and $n$-th column of the inverse matrix of $\Delta=H_{c}-(2 J \cos k) 1$ [125]. For the multi-port case, just replace $H_{c}$ with $H_{c}^{\prime}$ in $\Delta$.

We index -1 to $-\infty$ for sites of the left lead (lead $m$ ) and index 1 to $+\infty$ for sites of the right lead (lead $n$ ). The stationary states are the superpositions of incoming and outgoing waves 59]. The wavefunctions for the forward incidence $\psi_{L}^{k}(s)$ and backward incidence $\psi_{R}^{k}(s)$ are two linearly independent solutions

$$
\begin{aligned}
& \psi_{L}^{k}(s)=e^{i k s}+r_{L} e^{-i k s},(s<0) ; t_{L} e^{i k s},(s>0), \\
& \psi_{R}^{k}(s)=t_{R} e^{-i k s},(s<0) ; e^{-i k s}+r_{R} e^{i k s}(s>0) .
\end{aligned}
$$

The wavefunction continuity $\psi_{c, m}^{k}=\psi_{l, m}^{k}(0), \psi_{c, n}^{k}=$ $\psi_{l, n}^{k}(0)$ yields $\psi_{c, m}^{k}=1+r_{L}, \psi_{c, n}^{k}=t_{L}$ for the forward incidence; from Eq. (10), we have $\psi_{l, m}^{k}(-1)=e^{-i k}+r_{L} e^{i k}$, $\psi_{l, n}^{k}(1)=t_{L} e^{i k}$. Substituting these wavefunctions into Eqs. (8) and (9), we obtain $t_{L}$ and $r_{L}$. For the backward incidence, we have $\psi_{c, m}^{k}=t_{R}, \psi_{c, n}^{k}=1+r_{R}$; from Eq. (11), we have $\psi_{l, m}^{k}(-1)=t_{R} e^{i k}, \psi_{l, n}^{k}(1)=e^{-i k}+r_{R} e^{i k}$. Substituting these wavefunctions into Eqs. (8) and (9), we obtain $t_{R}$ and $r_{R}$. The scattering coefficients are

$$
\begin{aligned}
t_{L} & =\frac{\Delta_{n m}^{-1} J^{-1}\left(e^{i k}-e^{-i k}\right)}{\left(J^{-1}+\Delta_{m m}^{-1} e^{i k}\right)\left(J^{-1}+\Delta_{n n}^{-1} e^{i k}\right)-\Delta_{m n}^{-1} \Delta_{n m}^{-1} e^{2 i k}}, \\
r_{L} & =\frac{\Delta_{m n}^{-1} \Delta_{n m}^{-1}-\left(J^{-1} e^{i k}+\Delta_{m m}^{-1}\right)\left(J^{-1} e^{-i k}+\Delta_{n n}^{-1}\right)}{\left(J^{-1}+\Delta_{m m}^{-1} e^{i k}\right)\left(J^{-1}+\Delta_{n n}^{-1} e^{i k}\right)-\Delta_{m n}^{-1} \Delta_{n m}^{-1} e^{2 i k}}, \\
t_{R} & =\frac{\Delta_{m n}^{-1} J^{-1}\left(e^{i k}-e^{-i k}\right)}{\left(J^{-1}+\Delta_{m m}^{-1} e^{i k}\right)\left(J^{-1}+\Delta_{n n}^{-1} e^{i k}\right)-\Delta_{m n}^{-1} \Delta_{n m}^{-1} e^{2 i k}}, \\
r_{R} & =\frac{\Delta_{m n}^{-1} \Delta_{n m}^{-1}-\left(J^{-1} e^{i k}+\Delta_{n n}^{-1}\right)\left(J^{-1} e^{-i k}+\Delta_{m m}^{-1}\right)}{\left(J^{-1}+\Delta_{m m}^{-1} e^{i k}\right)\left(J^{-1}+\Delta_{n n}^{-1} e^{i k}\right)-\Delta_{m n}^{-1} \Delta_{n m}^{-1} e^{2 i k}} .
\end{aligned}
$$

The symmetric transmission is

$t_{L}=t_{R}$ for $\Delta_{m n}^{-1}=\Delta_{n m}^{-1} ;\left|t_{L}\right|=\left|t_{R}\right|$ for $\left|\Delta_{m n}^{-1}\right|=\left|\Delta_{n m}^{-1}\right|$.

The symmetric reflection is

$$
\begin{aligned}
& r_{L}=r_{R} \text { for } \Delta_{m m}^{-1}=\Delta_{n n}^{-1} \\
& \left|r_{L}\right|=\left|r_{R}\right| \text { for real } \Delta_{m m}^{-1}, \Delta_{n n}^{-1}, \Delta_{m n}^{-1} \Delta_{n m}^{-1} .
\end{aligned}
$$

The scattering properties of each pair of input-output leads are straightforwardly obtained in this manner. The symmetries of the scattering center $H_{c}$, imposing restrict constraints on the scattering coefficients, are essential to understand the symmetric scattering dynamics.

Symmetry protection. - The symmetry-protected scattering properties are closely related to the spatial structure of the scattering center and rely on two ways of mapping

$$
\mathbf{1}: U_{\mathbf{1}}|m(n)\rangle_{c} \rightarrow|m(n)\rangle_{c} ; \mathcal{I}: U_{\mathcal{I}}|m(n)\rangle_{c} \rightarrow|n(m)\rangle_{c},
$$

where $U_{\mathbf{1}, \mathcal{I}}=c, \mathbb{k}, q, p ;|m\rangle_{c}$ and $|n\rangle_{c}$ denote the two connection sites of the scattering center that are connected with the leads $m$ and $n$, respectively. The mapping manners reclassify eight even-parity symmetries

$$
C_{1}, C_{\mathcal{I}} ; K_{1}, K_{\mathcal{I}} ; Q_{1}, Q_{\mathcal{I}} ; P_{1}, P_{\mathcal{I}}
$$

TABLE I. Symmetry-protected constraint on the transmission and reflection for each individual symmetry.

\begin{tabular}{|c|c|c|c|c|}
\hline Symmetry & $C_{\mathbf{1}}, K_{\mathcal{I}}$ & $C_{\mathcal{I}}, K_{\mathbf{1}}$ & $Q_{\mathbf{1}}, P_{\mathcal{I}}$ & $Q_{\mathcal{I}}, P_{\mathbf{1}}$ \\
\hline Constraint & $\left|t_{L}\right|=\left|t_{R}\right|$ & $\left|r_{L}\right|=\left|r_{R}\right|$ & Both & None \\
\hline
\end{tabular}

The subscripts indicate the mapping manners. The $P_{\mathbf{1}}$ symmetry is trivial. The $P_{\mathcal{I}}$ symmetry is a generalized inversion symmetry and leads to symmetric transmission and reflection 36 43]. If the scattering center only has the $C_{\mathbf{1}}$ or $K_{\mathcal{I}}$ symmetry, the transmission is symmetric; but the reflection is asymmetric due to the lack of symmetry protection unless Eq. (14) is satisfied. Similarly, if the scattering center only has the $C_{\mathcal{I}}$ or $K_{1}$ symmetry, the reflection is symmetric, but the transmission is asymmetric unless Eq. (13) is satisfied. Table II lists the constraints for the corresponding symmetries (Supplemental Material A). For the scattering center without the $C_{\mathbf{1}}, K_{\mathcal{I}}, C_{\mathcal{I}}, K_{\mathbf{1}}, P_{\mathcal{I}}, Q_{\mathbf{1}}$ symmetries, both the transmission and reflection are asymmetric unless Eq. (13) or (14) is satisfied. The symmetry protection still valid even though the scattering coefficients diverge at the spectral singularity in the anomalous scattering [32, 126], where lasing occurs as the time-reversal process of perfect absorbing 8, 63]. $H_{c}=H_{c}^{T}$ belongs to the $C_{\mathbf{1}}$ symmetry and characterizes the Lorentz reciprocity in optics [2, 3, 33, 127]. The scattering center $H_{c}=\left[1, e^{-i \phi} ; e^{i \phi}, i\right]$ has the $C_{\mathbf{1}}$ symmetry, where the unitary operator is $c=\operatorname{diag}\left(1, e^{2 i \phi}\right)$ and the mapping between the connection sites is $c[|m\rangle,|n\rangle]^{T}=\left[|m\rangle, e^{2 i \phi}|n\rangle\right]^{T}$; consequently, $t_{L}=e^{2 i \phi} t_{R}$. The $C_{\mathcal{I}}$ symmetry is the combined $P_{\mathcal{I}} C_{\mathbf{1}}$ symmetry and leads to the symmetric reflection $r_{L}=r_{R}$ [128, 129]. The $K$ symmetry has the complex conjugation operation and relates to the time-reversal symmetry [98]. The $K_{1}$ symmetry results in the symmetric reflection $\left|r_{L}\right|=\left|r_{R}\right|$ [44, 45]. The $K_{\mathcal{I}}$ symmetry is the combined $P_{\mathcal{I}} K_{1}$ symmetry and results in the symmetric transmission $\left|t_{L}\right|=\left|t_{R}\right|$; the parity-time symmetry belongs to the $K_{\mathcal{I}}$ symmetry [45 95]. The $Q_{1}$ symmetry is a combined $C_{\mathbf{1}} K_{\mathbf{1}}$ or $C_{\mathcal{I}} K_{\mathcal{I}}$ symmetry. For example, $H_{c}=[0, i-1 ; i+1,1]$ has the $Q_{1}$ symmetry with $q=\sigma_{z}$; the Hermitian scattering centers possess the $Q_{1}$ symmetry with $q$ being the identity matrix. The transmission and reflection are both symmetric under the $Q_{1}$ symmetry protection [130]. In contrast, the combined $C_{\mathbf{1}} K_{\mathcal{I}}$ or $C_{\mathcal{I}} K_{1}$ symmetry: the $Q_{\mathcal{I}}$ symmetry, imposes no symmetric constraint on the scattering coefficients. For example, both the transmission and reflection are asymmetric for the $Q_{\mathcal{I}}$ symmetric non-Hermitian scattering center $H_{c}=\left[i \gamma, J e^{-\varphi} ; J e^{\varphi},-i \gamma\right]$, the corresponding unitary operator is $q=\sigma_{x}$; the cooperation between asymmetric coupling [131 134], gain, and loss destroys the symmetric transmission and reflection.

Breaking reciprocity.-Under the guidance of symmetry protection, we exemplify asymmetric light scatter- 

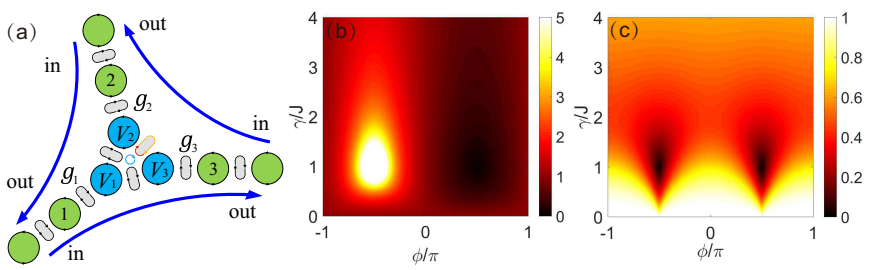

FIG. 2. (a) Schematic of the three-coupled-resonator scattering center. (b) $\left|t_{R}\right| /\left|t_{L}\right|$ for both $\{i \gamma,-i \gamma, 0\}$ and $\{0,-i \gamma, 0\}$. $\left|t_{R}\right| /\left|t_{L}\right|$ diverges at $\phi=-\pi / 2, \gamma / J=1$ and is cut to 5. (c) $\left|r_{R}\right| /\left|r_{L}\right|$ for $\{i \gamma,-i \gamma, 0\}$. (b, c) are for $g_{1}=g_{3}=J$ and $g_{2}=0$ (Supplemental Material B). The incidence has resonant frequency $\omega_{0}$.

ing in a three-coupled-resonator scattering center. In Fig. 2(a), the primary resonators (round-shape) are effectively coupled through the link resonators (stadiumshape). The Peierls phase factor $e^{ \pm i \phi}$ presents in one of the couplings between the central three resonators 135 137], where photons tunneling in the forward and backward directions experience different path lengths as indicated by the orange and red arrows in the link resonator [138, 139]. In the equations of motion [Eq. (6)], the scattering center is

$$
H_{c}=\left(\begin{array}{ccc}
V_{1} & J & J \\
J & V_{2} & J e^{-i \phi} \\
J & J e^{i \phi} & V_{3}
\end{array}\right)
$$

where $\omega_{0}+\operatorname{Re}\left(V_{j}\right)$ and $\operatorname{Im}\left(V_{j}\right)$ are the resonant frequency and gain/loss for the resonator $j=1,2,3$, respectively.

We take $g_{1}=g_{3}=J, g_{2}=0$ and discuss the symmetry protection. Without the gain and loss, the $Q_{\mathbf{1}}$ symmetry ensures the symmetric transmission and reflection. Without the nonreciprocal coupling, the $C_{\mathbf{1}}$ symmetry ensures the symmetric transmission. The interplay between the gain/loss and nonreciprocal coupling generates the asymmetric transmission. $H_{c}$ with complex $V_{1}=V_{3}^{*}$ and real $V_{2} \neq 0$ only has the $K_{\mathcal{I}}$ symmetry,

$$
\mathbb{k} H_{c}^{*} \mathbb{k}^{-1}=\left(\begin{array}{ccc}
V_{3}^{*} & J & J \\
J & V_{2}^{*} & J e^{-i \phi} \\
J & J e^{i \phi} & V_{1}^{*}
\end{array}\right), \mathbb{k}=\left(\begin{array}{ccc}
0 & 0 & 1 \\
0 & e^{-i \phi} & 0 \\
1 & 0 & 0
\end{array}\right) .
$$

Thus, the transmission is symmetric, but the reflection is asymmetric [63]. $H_{c}$ with $V_{1}=V_{3}$ and $V_{2} \neq V_{2}^{*}$ only has the $C_{\mathcal{I}}$ symmetry [140],

$$
c H_{c}^{T} c^{-1}=\left(\begin{array}{ccc}
V_{3} & J & J \\
J & V_{2} & J e^{-i \phi} \\
J & J e^{i \phi} & V_{1}
\end{array}\right), c=\left(\begin{array}{ccc}
0 & 0 & 1 \\
0 & e^{-i \phi} & 0 \\
1 & 0 & 0
\end{array}\right) .
$$

A single loss center $\left\{V_{1}, V_{2}, V_{3}\right\}=\{0,-i \gamma, 0\}$ has asymmetric transmission, but symmetric reflection; an ideal optical isolator with $S$-matrix $S=\left[r_{L}, t_{R} ; t_{L}, r_{R}\right]=$
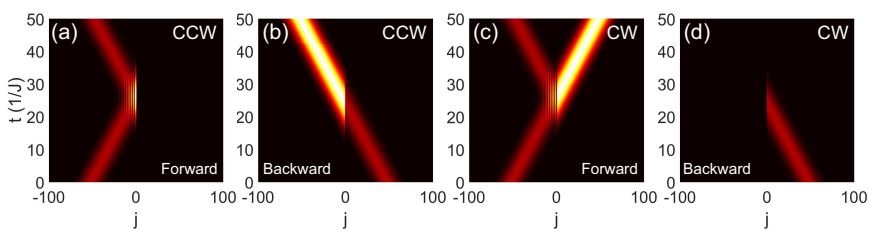

FIG. 3. Asymmetric scattering dynamics for Figs. 2(b, c) at $\gamma=J$. (a) Forward and (b) backward incidences for $\phi=$ $-\pi / 2$. The counterclockwise (CCW) mode and the clockwise (CW) mode of the resonator experience opposite magnetic fluxes. (c) Forward and (d) backward incidences for $\phi=\pi / 2$.

$\left(-i \sigma_{x} \pm \sigma_{y}\right) / 2$ is generated at $J=\gamma$ and $\phi=\mp \pi / 2$ for resonant incidence $k=-\pi / 2$ as indicated in Fig. 2 (b). In contrast, $\left|V_{1}\right| \neq\left|V_{3}\right|$ breaks all the symmetries of $H_{c}$ and both the transmission and reflection are asymmetric.

The striking asymmetric scattering for $\left\{V_{1}, V_{2}, V_{3}\right\}=$ $\{i \gamma,-i \gamma, 0\}$ in Figs. 2(b) and 2 (c) indicates a chiral perfect absorption [92] that unidirectional incidence is completely absorbed for the clockwise mode [73, 140, 141]. A unidirectional transmissionless $t_{L}=0, r_{L}=1 ; t_{R}=-2 i$, $r_{R}=0$ occurs at $\gamma=J, \phi=-\pi / 2$ [Figs. 3(a) and [3(b)] and a unidirectional absorption $t_{L}=-2 i, r_{L}=1$; $t_{R}=0, r_{R}=0$ occurs at $\gamma=J, \phi=\pi / 2$ [Figs. 3](c) and 3 (d)] for resonant incidence $k=-\pi / 2$.

For the three-port scattering, the transmission in the lead 3 (2) for incidence in the lead 1 is straightforwardly obtained from Eq. (12) by taking $m=1, n=3(2)$ and replacing $V_{2(3)}$ with $V_{2(3)}^{\prime}=V_{2(3)}+J e^{i k}$ in $H_{c}$ [124]. At $\left\{V_{1}, V_{2}, V_{3}\right\}=\{0,0,0\}$, the input resonantly outgoes from one of the adjacent leads as indicated by the blue arrows at $\phi=\pi / 2$ and inversely at $\phi=-\pi / 2$ for the resonant incidence, and functions as a circulator [111, 112, 121] with symmetric zero reflection protected by the $C_{\mathcal{I}}$ symmetry because $V_{2}^{\prime} \neq\left(V_{2}^{\prime}\right)^{*}$.

Without the gain and loss, the non-Hermitian dissipative coupling [113 115, 142 145] associated with nonreciprocal coupling can also break the symmetry protection and generate asymmetric transmission and reflection in the three-coupled-resonator scattering center

$$
H_{c}=\left(\begin{array}{ccc}
0 & -i \kappa & J \\
-i \kappa & 0 & J e^{-i \phi} \\
J & J e^{i \phi} & 0
\end{array}\right)
$$

More details are provided in Supplemental Material C.

Discussion.-For the two-port linear scattering center, the effective complex self-energy is absent. Thus, the non-Hermiticity is required to realize asymmetric transmission because Hermitian systems are $Q_{1}$ symmetry protected. The $C_{\mathbf{1}}, K_{\mathcal{I}}, P_{\mathcal{I}}, Q_{\mathbf{1}}$ symmetries should be absent to break symmetric transmission. In addition to the non-Hermiticity required to break the pseudo-Hermiticity ( $Q_{\mathbf{1}}$ symmetry), the nonreciprocal coupling is required to break the $C_{\mathbf{1}}$ symmetry protection. The simplest example is a two-site center with 
asymmetric coupling strengths $H_{c}=\left[0, J e^{-\varphi} ; J e^{\varphi}, 0\right]$ [128, 129]; the three-coupled-resonator scattering center with $\{0,-i \gamma, 0\}$ is another example, and other examples include systems studied in Refs. 7, 8, 110, 140, 141, 146148].

The $C_{\mathcal{I}}, K_{\mathbf{1}}, P_{\mathcal{I}}, Q_{\mathbf{1}}$ symmetries should be absent to break symmetric reflection. Provided that the resonator gain and/or loss are not balanced, all these four symmetries are absent. Thus, the asymmetric reflection ubiquitously presents in the intriguing scattering phenomena, including the unidirectional reflectionless [54, 55, 64, 68, 149], unidirectional lasing [8, 63], coherent perfect absorber laser [47, 49, 71, 150], chiral absorber [92], and chiral metamaterials [151, 152].

The situation $\left|V_{1}\right| \neq\left|V_{3}\right|$ in the three-coupledresonator (Fig. 3) and the systems studied in Refs. [8, 153] exemplify the asymmetric transmission and reflection without the protection of all the six symmetries $C_{\mathbf{1}}, C_{\mathcal{I}}, K_{\mathbf{1}}, K_{\mathcal{I}}, P_{\mathcal{I}}, Q_{\mathbf{1}}$. Properly incorporating nonreciprocal coupling, asymmetric coupling, dissipative coupling, gain, and loss generate asymmetric transmission and reflection.

For the multi-port scattering center, the effective scattering center $H_{c}^{\prime}$ may only possess the $C_{\mathbf{1}}, C_{\mathcal{I}}$ or $P_{\mathcal{I}}$ symmetry due to the momentum dependent self-energy in $H_{c}^{\prime}$. Thus, asymmetric scattering behavior easily occurs in the multi-port scattering center. If the leads are symmetrically coupled to the scattering center $H_{c}$, the transmission and (or) reflection of the multi-port scattering center are still symmetric under the $P_{\mathcal{I}}\left(C_{\mathbf{1}}\right.$ or $\left.C_{\mathcal{I}}\right)$ symmetry protection. Notably, the scattering properties of $H_{c}^{\prime}$ may be $K_{1}, K_{\mathcal{I}}$ or $Q_{1}$ symmetry-protected at certain momentum.

The symmetries with opposite parities, various symmetry types, and different ways of mapping may coexist in the scattering center. The constraints imposed by the symmetries on the scattering coefficients coexist and affect simultaneously. The symmetry protection provides fundamental guiding principles for manipulating quantum transport in mesoscopic and tailoring the light flow in the integrated photonics.

Conclusion.-We unveil the roles played by the symmetry for the scattering in non-Hermitian linear systems. The time-reversal symmetry, pseudo-Hermiticity (including Hermiticity), and generalized inversion symmetry protect the symmetric transmission and/or reflection (Table 【I); however, the particle-hole symmetry, chiral symmetry, and sublattice symmetry do not. These provide fundamental guiding principles for the light scattering in both Hermitian and non-Hermitian systems. Our findings are valid in the quantum systems and pave the way for further investigations on the transport in non-Hermitian physics.

We acknowledge the support of National Natural Science Foundation of China (Grants No. 11975128 and No. 11874225). L.J. acknowledges H. C. Wu for discus- sions and S. M. Zhang for useful comments on a first draft of this paper.

* jinliang@nankai.edu.cn

[1] C. W. J. Beenakker, Rev. Mod. Phys. 69, 732 (1997).

[2] R. J. Potton, Rep. Prog. Phys. 67, 717 (2004).

[3] D. L. Sounas and A. Alù, Nat. Photon. 11, 774 (2017).

[4] Z. Yu and S. Fan, Nat. Photon. 3, 91 (2009).

[5] L. Fan, J. Wang, L. T. Varghese, H. Shen, B. Niu, Y. Xuan, A. M. Weiner, and M. Qi, Science 335, 447 (2012).

[6] L. Chang, X. Jiang, S. Hua, C. Yang, J. Wen, L. Jiang, G. Li, G. Wang, and M. Xiao, Nat. Photon. 8, 524 (2014).

[7] X. Q. Li, X. Z. Zhang, G. Zhang, and Z. Song, Phys. Rev. A 91, 032101 (2015).

[8] L. Jin and Z. Song, Phys. Rev. Lett. 121, 073901 (2018).

[9] L. Du, Y. Zhang, and J.-H. Wu, Sci. Rep. 10, 1113 (2020).

[10] L. Bi, J. Hu, P. Jiang, D. H, Kim, G. F. Dionne, L. C. Kimerling, and C. A. Ross. Nat. Photon. 5, 758 (2011).

[11] H. Ramezani, Z. Lin, S. Kalish, T. Kottos, V. Kovanis, and I. Vitebskiy, Opt. Exp. 20, 26200 (2012).

[12] R. El-Ganainy, P. Kumar, and M. Levy, Opt. Lett. 38, 61 (2013).

[13] R. El-Ganainy, A. Eisfeld, M. Levy, and D. N. Christodoulides, Appl. Phys. Lett. 103, 161105 (2013).

[14] H. Ramezani, S. Kalish, I. Vitebskiy, and T. Kottos, Phys. Rev. Lett. 112, 043904 (2014).

[15] S. Lepri and G. Casati, Phys. Rev. Lett. 106, 164101 (2011).

[16] N. Bender, S. Factor, J. D. Bodyfelt, H. Ramezani, D. N. Christodoulides, F. M. Ellis, and T. Kottos, Phys. Rev. Lett. 110, 234101 (2013).

[17] Y. Shi, Z. Yu, and S. Fan, Nat. Photon. 9, 388 (2015).

[18] R. Huang, A. Miranowicz, J.-Q. Liao, F. Nori, and H. Jing, Phys. Rev. Lett. 121, 153601 (2018).

[19] D. L. Sounas and A. Alù, Phys. Rev. Lett. 118, 154302 (2017).

[20] K. Fang, Z. Yu, and S. Fan, Phys. Rev. Lett. 108, 153901 (2012).

[21] K. Fang, Z. Yu, and S. Fan, Nat. Photon. 6, 782 (2012).

[22] M. C. Rechtsman, J. M. Zeuner, Y. Plotnik, Y. Lumer, D. Podolsky, F. Dreisow, S. Nolte, M. Segev, and A. Szameit, Nature 496, 196 (2013).

[23] L. D. Tzuang, K. Feng, P. Nussenzveig, S. Fan, and M. Lipson, Nat. Photon. 8, 701 (2014).

[24] E. Li, B. J. Eggleton, K. Fang, and S. Fan, Nat. Commun. 5, 3225 (2014).

[25] S. Longhi, Opt. Lett. 39, 5892 (2014).

[26] S. Mukherjee, M. Di Liberto, P. Öhberg, R. R. Thomson, and N. Goldman, Phys. Rev. Lett. 121, 075502 (2018).

[27] N. R. Cooper, J. Dalibard, and I. B. Spielman, Rev. Mod. Phys. 91, 015005 (2019).

[28] T. Ozawa, H. M. Price, A. Amo, N. Goldman, M. Hafezi, L. Lu, M. Rechtsman, D. Schuster, J. Simon, O. Zilberberg, and I. Carusotto, Rev. Mod. Phys. 91, 015006 (2019).

[29] Y. Lumer, M. A. Bandres, M. Heinrich, L. J. 
Maczewsky, H. Herzig-Sheinfux, A. Szameit, and M. Segev, Nat. Photon. 13, 339 (2019).

[30] M. Kremer, I. Petrides, E. Meyer, M. Heinrich, O. Zilberberg, and A. Szameit, Nat. Commun. 11, 907 (2020).

[31] A. Dutt, Q. Lin, L. Yuan, M. Minkov, M. Xiao, and S. Fan, Science 367, 59 (2020).

[32] A. Krasnok, D. Baranov, H. Li, M.-A. Miri, F. Monticone, and A. Alù, Adv. Opt. Photon. 11, 892 (2019).

[33] D. Jalas, A. Petrov, M. Eich, W. Freude, S. Fan, Z. Yu, R. Baets, M. Popović, A. Melloni, J. D. Joannopoulos, M. Vanwolleghem, C. R. Doerr, and H. Renner, Nat. Photon. 7, 579 (2013).

[34] S. Fan, R. Baets, A. Petrov, Z. Yu, J. D. Joannopoulos, W. Freude, A. Melloni, M. Popović, M. Vanwolleghem, D. Jalas, M. Eich, M. Krause, H. Renner, E. Brinkmeyer, and C. R. Doerr, Science 335, 38 (2012).

[35] X. Yin and X. Zhang, Nat. Mater. 12, 175 (2013).

[36] Y. D. Chong, L. Ge, H. Cao, and A. D. Stone, Phys. Rev. Lett. 105, 053901 (2010).

[37] W. Wan, Y. Chong, L. Ge, H. Noh, A. D. Stone, and H. Cao, Science 331, 889 (2011).

[38] D. G. Baranov, A. Krasnok, T. Shegai, A. Alù, and Y. Chong, Nat. Rev. Mater. 2, 17064 (2017).

[39] D. G. Baranov, A. Krasnok, and A. Alù, Optica 4, 1457 (2017).

[40] S. Longhi, Opt. Lett. 43, 2122 (2018).

[41] G. Trainiti, Y. Ra'di, M. Ruzzene, and A. Alù, Sci. Adv. 5, eaaw3255 (2019).

[42] Q. Zhong, L. Simonson, T. Kottos, and R. El-Ganainy, Phys. Rev. Research 2, 013362 (2020).

[43] P. C. Burke, J. Wiersig, and M. Haque, Phys. Rev. A 102, 012212 (2020).

[44] F. Cannata, J.-P. Dedonder, and A. Ventura, Ann. Phys. (NY) 322, 397 (2007).

[45] L. Jin, X. Z. Zhang, G. Zhang, and Z. Song, Sci. Rep. 6, 20976 (2016).

[46] J. G. Muga, J. P. Palao, B. Navarro, and I. L. Egusquiza, Phys. Rep. 395, 357 (2004).

[47] S. Longhi, Phys. Rev. A 82, 031801(R) (2010).

[48] S. Longhi, J. Phys. A: Math. Theor. 44, 485302 (2011).

[49] Y. D. Chong, L. Ge, and A. D. Stone, Phys. Rev. Lett. 106, 093902 (2011).

[50] Z. Lin, H. Ramezani, T. Eichelkraut, T. Kottos, H. Cao, and D. N. Christodoulides, Phys. Rev. Lett. 106, 213901 (2011).

[51] L. Feng, M. Ayache, J. Huang, Y.-L. Xu, M.-H. Lu, Y.F. Chen, Y. Fainman, and A. Scherer, Science 333, 729 (2011).

[52] S. Kalish, Z. Lin, and T. Kottos, Phys. Rev. A 85, 055802 (2012).

[53] L. Ge, Y. D. Chong, and A. D. Stone, Phys. Rev. A 85, 023802 (2012).

[54] A. Regensburger, C. Bersch, M.-A. Miri, G. Onishchukov, D. N. Christodoulides, and U. Peschel, Nature 488, 167 (2012).

[55] L. Feng, Y.-L. Xu, W. S. Fegadolli, M.-H. Lu, J. E. B. Oliveira, V. R. Almeida, Y.-F. Chen, and A. Scherer, Nat. Mater. 12, 108 (2013).

[56] G. Castaldi, S. Savoia, V. Galdi, A. Alù, and N. Engheta, Phys. Rev. Lett. 110, 173901 (2013).

[57] H. Schomerus, Phil. Trans. R. Soc. A 371, 20120194 (2013).

[58] Z. Ahmed, Phys. Lett. A 377, 957 (2013).

[59] P. Ambichl, K. G. Makris, L. Ge, Y. Chong, A. D. Stone, and S. Rotter, Phys. Rev. X 3, 041030 (2013).

[60] S. Savoia, G. Castaldi, and V. Galdi, Phys. Rev. B 89 , 085105 (2014).

[61] A. Mostafazadeh, J. Phys. A: Math. Theor. 47, 505303 (2014).

[62] J.-H. Wu, M. Artoni, and G. C. La Rocca, Phys. Rev. Lett. 113, 123004 (2014).

[63] H. Ramezani, H.-K. Li, Y. Wang, and X. Zhang, Phys. Rev. Lett. 113, 263905 (2014).

[64] R. Fleury, D. Sounas, and A. Alù, Nat. Commun. 6, 5905 (2015).

[65] J. Gear, F. Liu, S. T. Chu, S. Rotter, and J. Li, Phys. Rev. A 91, 033825 (2015).

[66] Y. Huang, G. Veronis, and C. Min, Opt. Exp. 23, $029882(2015)$.

[67] M.-A. Miri, M. A. Eftekhar, M. Facao, A. F. Abouraddy, A. Bakry, M. A. N. Razvi, A. Alshahrie, A. Alù, and D. N. Christodoulides, J. Opt. 18, 075104 (2016).

[68] H. Zhao, W. S. Fegadolli, J. Yu, Z. Zhang, L. Ge, A. Scherer, and L. Feng, Phys. Rev. Lett. 117, 193901 (2016).

[69] J.-H. Wu, M. Artoni, and G. C. La Rocca, Sci. Rep. 6, 35356 (2016).

[70] L. Ge and L. Feng, Phys. Rev. A 94, 043836 (2016).

[71] Z. J. Wong, Y. Xu, J. Kim, K. O. Brien, Y. Wang, L. Feng, and X. Zhang, Nat. Photon. 10, 796 (2016).

[72] J. Christensen, M. Willatzen, V. R. Velasco, and M.-H. Lu, Phys. Rev. Lett. 116, 207601 (2016).

[73] H. Ramezani, Y. Wang, E. Yablonovitch, and X. Zhang, IEEE J. Sel. Top. Quantum Electron. 22, 115 (2016).

[74] S. Xiao, J. Gear, S. Rotter, and J. Li, New J. Phys. 18, 085004 (2016).

[75] A. Mostafazadeh, J. Phys. A: Math. Theor. 49, 445302 (2016).

[76] J. Gear, Y. Sun, S. Xiao, L. Zhang, R. Fitzgerald, S. Rotter, H. Chen, and J. Li, New J. Phys. 19, 123041 (2017).

[77] J.-H. Wu, M. Artoni, and G. C. La Rocca, Phys. Rev. A 95, 053862 (2017).

[78] L. Ge and L. Feng, Phys. Rev. A 95, 013813 (2017).

[79] P. A. Kalozoumis, C. V. Morfonios, G. Kodaxis, F. K. Diakonos, and P. Schmelcher, Appl. Phys. Lett. 110, 121106 (2017).

[80] L.-L. Zhang, G.-H. Zhan, Z.-Z. Li, and W.-J. Gong, Phys. Rev. A 96, 062133 (2017).

[81] L. Jin, Phys. Rev. A 97, 033840 (2018).

[82] J. Luo, J. Li, and Y. Lai, Phys. Rev. X 8, 031035 (2018).

[83] F. Gao, J.-R. Dong, Y.-M. Liu, and Y. Zhang J. Opt. Soc. Am. B 35, 2075 (2018).

[84] T. T. Koutserimpas, A. Alù, and R. Fleury, Phys. Rev. A 97, 013839 (2018).

[85] C. Shen, J. Li, X. Peng, and S. A. Cummer, Phys. Rev. Mater. 2, 125203 (2018).

[86] N. Wu, C. Zhang, X. R. Jin, Y. Q. Zhang, and Y. P. Lee, Opt. Exp. 26, 3839 (2018).

[87] A. Merkel, V. Romero-García, J.-P. Groby, J. Li, and J. Christensen, Phys. Rev. B 98, 201102(R) (2018).

[88] Y. Zhi, X. Yang, J. Wu, S. Du, P. Cao, D. Deng, and C. T. Liu, Photon. Res. 6, 579 (2018).

[89] H. Wu, X. Yang, Y. Tang, X. Tang, D. Deng, H. Liu, and Z. Wei, Ann. Phys. (Berlin) 531, 1900120 (2019).

[90] M. A. Simón, A. Buendía, A. Kiely, A. Mostafazadeh, and J. G. Muga, Phys. Rev. A 99, 052110 (2019). 
[91] Z. Zhao, C. Guo, and S. Fan, Phys. Rev. A 99, 033839 (2019).

[92] W. R. Sweeney, C. W. Hsu, S. Rotter, and A. D. Stone, Phys. Rev. Lett. 122, 093901 (2019).

[93] W. R. Sweeney, C. W. Hsu, and A. D. Stone, Phys. Rev. A, 102, 063511 (2020).

[94] A. Novitsky, D. Lyakhov, Dominik Michels, A. A. Pavlov, A. S. Shalin, and D. V. Novitsky, Phys. Rev. A 101, 043834 (2020).

[95] H. Zhu, X. Yang, Z. Lin, X. Liu, X. Yang, Opt. Commun. 459, 124945 (2020).

[96] S. Rotter and S. Gigan, Rev. Mod. Phys. 89, 015005 (2017).

[97] K. Kawabata, S. Higashikawa, Z. Gong, Y. Ashida, and M. Ueda, Nat. Commun. 10, 297 (2019).

[98] K. Kawabata, K. Shiozaki, M. Ueda, and M. Sato, Phys. Rev. X 9, 041015 (2019).

[99] V. V. Konotop, J. Yang, and D. A. Zezyulin, Rev. Mod. Phys. 88, 035002 (2016).

[100] S. V. Suchkov, A. A. Sukhorukov, J. Huang, S. V. Dmitriev, C. Lee, and Y. S. Kivshar, Laser Photon. Rev. 10, 177 (2016)

[101] L. Feng, R. El-Ganainy, and L. Ge, Nat. Photon. 11, 752 (2017).

[102] S. Longhi, Europhys. Lett. 120, 64001 (2017).

[103] R. El-Ganainy, K. G. Makris, M. Khajavikhan, Z. H. Musslimani, S. Rotter, and D. N. Christodoulides, Nat. Phys. 14, 11 (2018).

[104] M.-A. Miri and A. Alù, Science 363, eaar7709 (2019).

[105] S. K. Özdemir, S. Rotter, F. Nori, and L. Yang, Nat. Mater. 18, 783 (2019).

[106] S. K. Gupta, Y. Zou, X.-Y. Zhu, M.-H. Lu, L.-J. Zhang, X.-P. Liu, and Y.-F. Chen, Adv. Mater. 32, 1903639 (2019).

[107] D. Bernard and A. LeClair, arXiv:cond-mat/0110649 (2001).

[108] We emphasize two aspects in the definitions of symmetries that distinct from the topological classification [98]:

(i) the signs $\epsilon_{\mathbb{k}}$ and $\epsilon_{q}$ in the $K$ and $Q$ symmetries; which are absent in the definitions of the $K$ and $Q$ symmetries for topological classification because $H_{c}$ and $i H_{c}$ have identical topological properties. However, the scattering properties of $H_{c}$ and $i H_{c}$ are not identical. The symmetries with opposite parities impose different scattering features; (ii) the even-parity $P$ symmetry.

[109] The combination of two symmetries that belong to the same type gives the $P$ symmetry. The combination of the $P$ symmetry and any of the $C, K, Q$ symmetries does not alter the $C, K, Q$ symmetries. The combination of any two of the three symmetries $C, K, Q$ produces the third symmetry.

[110] W. Gou, T. Chen, D. Xie, T. Xiao, T.-S. Deng, B. Gadway, W. Yi, and B. Yan, Phys. Rev. Lett. 124, 070402 (2020).

[111] R. Fleury, D. L. Sounas, C. F. Sieck, M. R. Haberman, and A. Alù, Science 343, 516 (2014).

[112] K. M. Sliwa, M. Hatridge, A. Narla, S. Shankar, L. Frunzio, R. J. Schoelkopf, and M. H. Devoret, Phys. Rev. X 5, 041020 (2015).

[113] F. Yang, Y.-C. Liu, and L. You, Phys. Rev. A 96, 053845 (2017).

[114] H. C. Wu, X. M. Yang, L. Jin, and Z. Song, Phys. Rev. B 102, 161101(R) (2020).
[115] V. V. Konotop and D. A. Zezyulin, Phys. Rev. Lett. 120, 123902 (2018).

[116] A. Müllers, B. Santra, C. Baals, J. Jiang, J. Benary, R. Labouvie, D. A. Zezyulin, V. V. Konotop, and H. Ott, Sci. Adv. 4, eaat6539 (2018).

[117] H. Ramezani, P. K. Jha, Y. Wang, and X. Zhang, Phys. Rev. Lett. 120, 043901 (2018).

[118] H. A. Haus and W. P. Huang, Proc. IEEE 79, 1505 (1991).

[119] S. Fan, W. Suh, and J. D. Joannopoulos, J. Opt. Soc. Am. A 20, 569 (2003).

[120] J. D. Joannopoulos, S. G. Johnson, J. N. Winn, and R. D. Meade, Photonic Crystals: Molding the Flow of Light (Princeton University Press, Princeton, NJ, 2008).

[121] N. A. Estep, D. L. Sounas, J. Soric, and A. Alù, Nat. Phys. 10, 923 (2014).

[122] L. Jin and Z. Song, Phys. Rev. A 81, 022107 (2010).

[123] L. Jin and Z. Song, Phys. Rev. A 81, 032109 (2010).

[124] L. Jin and Z. Song, J. Phys. A: Math. Theor. 44, 375304 (2011).

[125] If the determint $|\Delta|$ of matrix $\Delta$ vanishes, just regarding the inverse matrix $\Delta^{-1}$ as the adjoint matrix $\Delta^{\text {adj }}$ of matrix $\Delta$ in Eqs. (13) and (14). They satisfies the relation $\Delta^{-1}=\Delta^{\text {adj }} /|\Delta|$.

[126] P. Wang, L. Jin, G. Zhang, and Z. Song, Phys. Rev. A 94, 053834 (2016).

[127] L. Jin, Phys. Rev. A 98, 022117 (2018).

[128] X. Z. Zhang and Z. Song, Ann. Phys. (NY) 339, 109 (2013).

[129] C. Li, L. Jin, and Z. Song, Phys. Rev. A 95, 022125 (2017).

[130] L. Jin and Z. Song, Phys. Rev. A 85, 012111 (2012).

[131] S. Malzard, C. Poli, and H. Schomerus, Phys. Rev. Lett. 115, 200402 (2015).

[132] S. Longhi, D. Gatti, and G. Della Valle, Phys. Rev. B 92, 094204 (2015).

[133] J. Ren, Y. G. N. Liu, M. Parto, W. E. Hayenga, M. P. Hokmabadi, D. N. Christodoulides, and M. Khajavikhan, Opt. Exp. 26, 27153 (2018).

[134] L. Jin and Z. Song, Phys. Rev. B 99, 081103(R) (2019).

[135] C. W. Peterson, S. Kim, J. T. Bernhard, and G. Bahl, Sci. Adv. 4, eaat0232 (2018).

[136] L. Jin and Z. Song, Phys. Rev. A 93, 062110 (2016).

[137] L. Jin, Phys. Rev. A 97, 012121 (2018).

[138] M. Hafezi, S. Mittal, J. Fan, A. Migdall, and J. M. Taylor, Nat. Photon. 7, 1001 (2013).

[139] M. Hafezi, Phys. Rev. Lett. 112, 210405 (2014).

[140] L. Jin, P. Wang, and Z. Song, Sci. Rep. 6, 32919 (2016).

[141] S. Longhi, Opt. Lett. 40, 1278 (2015).

[142] P. Peng, W. Cao, C. Shen, W. Qu, J. Wen, L. Jiang, and Y. Xiao, Nat. Phys. 12, 1139 (2016).

[143] Y. Li, Y.-G. Peng, L. Han, M.-A. Miri, W. Li, M. Xiao, X.-F. Zhu, J. Zhao, A. Alù, S. Fan, and C.-W. Qiu, Science 364, 170 (2019).

[144] Y. Jiang, Y. Mei, Y. Zuo, Y. Zhai, J. Li, J. Wen, and S. Du, Phys. Rev. Lett. 123, 193604 (2019).

[145] Y.-P. Wang and C.-M. Hu, J. Appl. Phys. 127, 130901 (2020).

[146] C. He, X.-L. Zhang, L. Feng, M.-H. Lu, and Y.-F. Chen, Appl. Phys. Lett. 99, 151112 (2011).

[147] X.-F. Li, X. Ni, L. Feng, M.-H. Lu, C. He, and Y.-F. Chen, Phys. Rev. Lett. 106, 084301 (2011).

[148] Y.-L. Xu, L. Feng, M.-H. Lu, Y.-F. Chen, Phys. Lett. 
A 376, 886 (2012).

[149] Y. Huang, Y. Shen, C. Min, S. Fan, and G. Veronis, Nanophotonics 6, 977 (2017).

[150] S. Cao and Z. Hou, Phys. Rev. Appl. 12, 064016 (2019).

[151] S. Droulias, I. Katsantonis, M. Kafesaki, C. M. Soukoulis, and E. N. Economou, Phys. Rev. Lett. 122, 213201 (2019).

[152] I. Katsantonis, S. Droulias, C. M. Soukoulis, E. N. Economou, and M. Kafesaki, Photonics 7, 43 (2020).

[153] L. Jin, P. Wang and Z. Song, New J. Phys. 19, 015010 (2017). 


\title{
SUPPLEMENTAL MATERIAL FOR "SYMMETRY-PROTECTED SCATTERING IN NON-HERMITIAN LINEAR SYSTEMS"
}

\author{
L. Jin ${ }^{1, *}$ and Z. Song ${ }^{1}$ \\ ${ }^{1}$ School of Physics, Nankai University, Tianjin 300071, China
}

\section{A: Proof of the constraints on the scattering coefficients for discrete symmetries}

Symmetric scattering coefficients. - The transmission and reflection coefficients for the input-output in the leads $m$ and $n$ are

$$
\begin{aligned}
t_{L} & =\frac{\Delta_{n m}^{-1} J^{-1}\left(e^{i k}-e^{-i k}\right)}{\left(J^{-1}+\Delta_{m m}^{-1} e^{i k}\right)\left(J^{-1}+\Delta_{n n}^{-1} e^{i k}\right)-\Delta_{m n}^{-1} \Delta_{n m}^{-1} e^{2 i k}}, \\
r_{L} & =\frac{\Delta_{m n}^{-1} \Delta_{n m}^{-1}-\left(J^{-1} e^{i k}+\Delta_{m m}^{-1}\right)\left(J^{-1} e^{-i k}+\Delta_{n n}^{-1}\right)}{\left(J^{-1}+\Delta_{m m}^{-1} e^{i k}\right)\left(J^{-1}+\Delta_{n n}^{-1} e^{i k}\right)-\Delta_{m n}^{-1} \Delta_{n m}^{-1} e^{2 i k}} . \\
t_{R} & =\frac{\Delta_{m n}^{-1} J^{-1}\left(e^{i k}-e^{-i k}\right)}{\left(J^{-1}+\Delta_{m m}^{-1} e^{i k}\right)\left(J^{-1}+\Delta_{n n}^{-1} e^{i k}\right)-\Delta_{m n}^{-1} \Delta_{n m}^{-1} e^{2 i k}}, \\
r_{R} & =\frac{\Delta_{m n}^{-1} \Delta_{n m}^{-1}-\left(J^{-1} e^{i k}+\Delta_{n n}^{-1}\right)\left(J^{-1} e^{-i k}+\Delta_{m m}^{-1}\right)}{\left(J^{-1}+\Delta_{m m}^{-1} e^{i k}\right)\left(J^{-1}+\Delta_{n n}^{-1} e^{i k}\right)-\Delta_{m n}^{-1} \Delta_{n m}^{-1} e^{2 i k}},
\end{aligned}
$$

where $\Delta=H_{c}+\left(\omega_{0}-\omega\right) \mathbf{1}=H_{c}-(2 J \cos k) \mathbf{1}, \Delta^{-1}$ indicates the inverse of $\Delta$, and $\Delta_{m n}^{-1}$ indicates the element on the $m$-th row and the $n$-th column of $\Delta^{-1}$.

It is obvious that

$$
\begin{aligned}
& t_{L}=t_{R} \text { for } \Delta_{n m}^{-1}=\Delta_{m n}^{-1}, \\
& r_{L}=r_{R} \text { for } \Delta_{m m}^{-1}=\Delta_{n n}^{-1} .
\end{aligned}
$$

Alternatively,

$$
\left|t_{L}\right|^{2}=\left|t_{R}\right|^{2} \text { for }\left|\Delta_{n m}^{-1}\right|=\left|\Delta_{m n}^{-1}\right| \text {. }
$$

The difference between the numerators of $\left|r_{L}\right|^{2}$ and $\left|r_{R}\right|^{2}$ is given by

$$
\begin{aligned}
& \left|\Delta_{m n}^{-1} \Delta_{n m}^{-1}-\left(J^{-1} e^{i k}+\Delta_{m m}^{-1}\right)\left(J^{-1} e^{-i k}+\Delta_{n n}^{-1}\right)\right|^{2}-\left|\Delta_{m n}^{-1} \Delta_{n m}^{-1}-\left(J^{-1} e^{i k}+\Delta_{n n}^{-1}\right)\left(J^{-1} e^{-i k}+\Delta_{m m}^{-1}\right)\right|^{2} \\
= & 2 J^{-2} i \sin (2 k)\left(\Delta_{n n}^{-1} \Delta_{m m}^{-1 *}-\Delta_{m m}^{-1} \Delta_{n n}^{-1 *}\right) \\
& +2 J^{-1} i \sin k\left[\left(J^{-2}+\Delta_{m m}^{-1 *} \Delta_{n n}^{-1 *}-\Delta_{m n}^{-1 *} \Delta_{n m}^{-1 *}\right)\left(\Delta_{n n}^{-1}-\Delta_{m m}^{-1}\right)-\left(J^{-2}+\Delta_{m m}^{-1} \Delta_{n n}^{-1}-\Delta_{m n}^{-1} \Delta_{n m}^{-1}\right)\left(\Delta_{n n}^{-1 *}-\Delta_{m m}^{-1 *}\right)\right] .
\end{aligned}
$$

Thus, we have $\left|r_{L}\right|^{2}=\left|r_{R}\right|^{2}$ for real $\Delta_{m m}^{-1}, \Delta_{n n}^{-1}$, and $\Delta_{m n}^{-1} \Delta_{n m}^{-1}$. These are the conclusions of Eqs. (9) and (10) in the main text. Notably, the accidental symmetric reflection $\left|r_{L}\right|^{2}=\left|r_{R}\right|^{2}$ occurs if the conditions $J^{-2}+\Delta_{m m}^{-1 *} \Delta_{n n}^{-1 *}-$ $\Delta_{m n}^{-1 *} \Delta_{n m}^{-1 *}=0$ and $\Delta_{n n}^{-1} \Delta_{m m}^{-1 *}=\Delta_{m m}^{-1} \Delta_{n n}^{-1 *}$ are simultaneously satisfied.

Symmetry-protected scattering.-The mapping relations are

$$
\begin{aligned}
& \mathbf{1}: U_{\mathbf{1}}\left[\cdots,|m\rangle_{c}, \cdots,|n\rangle_{c}, \cdots\right]^{T}=\left[\cdots,|m\rangle_{c}, \cdots, e^{i \alpha}|n\rangle_{c}, \cdots\right]^{T}, \\
& \mathcal{I}: U_{\mathcal{I}}\left[\cdots,|m\rangle_{c}, \cdots,|n\rangle_{c}, \cdots\right]^{T}=\left[\cdots,|n\rangle_{c}, \cdots, e^{i \alpha}|m\rangle_{c}, \cdots\right]^{T} .
\end{aligned}
$$

In the general situation, the phase factor $e^{i \alpha}$ is not necessarily to be 1 . The four elements in the unitary operators that relate to the mapping between the connection sites $|m\rangle_{c}$ and $|n\rangle_{c}$ are

$$
\begin{aligned}
& \mathbf{1}:\left(\begin{array}{cc}
U_{\mathbf{1}, m m} & U_{\mathbf{1}, n m} \\
U_{\mathbf{1}, m n} & U_{\mathbf{1}, n n}
\end{array}\right)=\left(\begin{array}{cc}
1 & 0 \\
0 & e^{i \alpha}
\end{array}\right), \\
& \mathcal{I}:\left(\begin{array}{cc}
U_{\mathcal{I}, m m} & U_{\mathcal{I}, n m} \\
U_{\mathcal{I}, m n} & U_{\mathcal{I}, n n}
\end{array}\right)=\left(\begin{array}{cc}
0 & 1 \\
e^{i \alpha} & 0
\end{array}\right) .
\end{aligned}
$$

Other relevant elements in the mapping relation are $U_{\mathbf{1}, m j}=0$ and $U_{\mathbf{1}, j n}=0$ for $j \neq m, n ; U_{\mathcal{I}, m j}=0$ and $U_{\mathcal{I}, j n}=0$ for $j \neq m, n$. 
The scattering center with the even-parity $C$ symmetry satisfies $H_{c}=c H_{c}^{T} c^{-1}$. From the definition $\Delta=H_{c}-$ $(2 J \cos k) \mathbf{1}$, we have the relation $\Delta=c \Delta^{T} c^{-1}$; therefore, we obtain

$$
\Delta^{-1}=c\left(\Delta^{-1}\right)^{T} c^{-1} .
$$

For the $C_{\mathbf{1}}$ symmetry, we have

$$
\Delta_{n m}^{-1}=e^{i \alpha} \Delta_{m n}^{-1}
$$

thus, we obtain the symmetric transmission

$$
\left|t_{L}\right|=\left|t_{R}\right|
$$

For the $C_{\mathcal{I}}$ symmetry, we have

$$
\Delta_{m m}^{-1}=\Delta_{n n}^{-1}
$$

besides, the $C_{\mathcal{I}}$ symmetry also demands $e^{i \alpha}=1$ or $\Delta_{m n}^{-1}=\Delta_{n m}^{-1}=0$. Thus, in general case we only have the symmetric reflection

$$
r_{L}=r_{R}
$$

The scattering center with the even-parity $K$ symmetry satisfies $H_{c}=\mathbb{k}_{c}^{*} \mathbb{k}^{-1}$. Therefore, we obtain the relation $\Delta=\mathbb{k} \Delta^{*} \mathbb{k}^{-1} ;$ and consequently,

$$
\Delta^{-1}=\mathbb{k}\left(\Delta^{-1}\right)^{*} \mathbb{k}^{-1}
$$

For the $K_{1}$ symmetry, we obtain

$$
\Delta_{m m}^{-1}=\left(\Delta_{m m}^{-1}\right)^{*}, \Delta_{n n}^{-1}=\left(\Delta_{n n}^{-1}\right)^{*} ; \Delta_{n m}^{-1}=e^{i \alpha}\left(\Delta_{n m}^{-1}\right)^{*}, \Delta_{m n}^{-1}=e^{-i \alpha}\left(\Delta_{m n}^{-1}\right)^{*},
$$

thus, $\Delta_{m m}^{-1}, \Delta_{n n}^{-1}$, and $\Delta_{m n}^{-1} \Delta_{n m}^{-1}$ are all real numbers; and we have symmetric reflection

$$
\left|r_{L}\right|=\left|r_{R}\right| \text {. }
$$

For the $K_{\mathcal{I}}$ symmetry, we obtain

$$
\Delta_{n m}^{-1}=e^{i \alpha}\left(\Delta_{m n}^{-1}\right)^{*},
$$

thus, we have symmetric transmission

$$
\left|t_{L}\right|=\left|t_{R}\right|
$$

we also have $\Delta_{m m}^{-1}=\left(\Delta_{n n}^{-1}\right)^{*}$, which does not lead to a symmetric relation on the scattering coefficients.

The scattering center with the even-parity $Q$ symmetry satisfies $H_{c}=q H_{c}^{\dagger} q^{-1}$. Therefore, we obtain $\Delta=q \Delta^{\dagger} q^{-1}$; and consequently

$$
\Delta^{-1}=q\left(\Delta^{-1}\right)^{\dagger} q^{-1}
$$

For the $Q_{1}$ symmetry, we have

$$
\Delta_{m m}^{-1}=\left(\Delta_{m m}^{-1}\right)^{*}, \Delta_{n n}^{-1}=\left(\Delta_{n n}^{-1}\right)^{*} ; \Delta_{n m}^{-1}=e^{i \alpha}\left(\Delta_{m n}^{-1}\right)^{*}, \Delta_{m n}^{-1}=e^{-i \alpha}\left(\Delta_{n m}^{-1}\right)^{*},
$$

the $Q_{1}$ symmetry also requires $e^{2 i \alpha}=1$. From $\Delta_{n m}^{-1}=e^{i \alpha}\left(\Delta_{m n}^{-1}\right)^{*}$, we obtain $\left|t_{L}\right|=\left|t_{R}\right|$. Notice that $\Delta_{m m}^{-1}, \Delta_{n n}^{-1}$, and $\Delta_{m n}^{-1} \Delta_{n m}^{-1}$ are all real numbers; thus, we have both the symmetric transmission and reflection

$$
\left|t_{L}\right|=\left|t_{R}\right|,\left|r_{L}\right|=\left|r_{R}\right|
$$

For the $Q_{\mathcal{I}}$ symmetry, we have

$$
\Delta_{m m}^{-1}=\left(\Delta_{n n}^{-1}\right)^{*}, \Delta_{n m}^{-1}=e^{i \alpha}\left(\Delta_{n m}^{-1}\right)^{*}, \Delta_{m n}^{-1}=e^{-i \alpha}\left(\Delta_{m n}^{-1}\right)^{*} .
$$


These constraints are insufficient to result in the symmetric transmission as well as the symmetric reflection.

The scattering center with the even-parity $P$ symmetry satisfies $H_{c}=p H_{c} p^{-1}$; therefore, we obtain $\Delta=p \Delta p^{-1}$ and

$$
\Delta^{-1}=p\left(\Delta^{-1}\right) p^{-1}
$$

For the $P_{1}$ symmetry, we have

$$
\Delta_{n m}^{-1}=e^{i \alpha} \Delta_{n m}^{-1}, \Delta_{m n}^{-1}=e^{-i \alpha} \Delta_{m n}^{-1}
$$

These constraints are insufficient to result in the symmetric transmission as well as the symmetric reflection.

For the $P_{\mathcal{I}}$ symmetry, we have

$$
\Delta_{m m}^{-1}=\Delta_{n n}^{-1}, \Delta_{n m}^{-1}=e^{i \alpha} \Delta_{m n}^{-1} .
$$

Thus, we have both the symmetric transmission and reflection

$$
\left|t_{L}\right|=\left|t_{R}\right|, r_{L}=r_{R}
$$

These conclusions are summarized in Supplemental Table II and give the symmetric constraints on the scattering coefficients listed in Table I of the main text.

TABLE II. Symmetry-protected constraints on the transmission and reflection coefficients.

\begin{tabular}{|c|c|c|c|c|c|c|c|}
\hline Symmetry & $C_{\mathbf{1}}$ & $C_{\mathcal{I}}$ & $K_{\mathbf{1}}$ & $K_{\mathcal{I}}$ & $Q_{\mathbf{1}}$ & $P_{\mathcal{I}}$ & $Q_{\mathcal{I}}, P_{\mathbf{1}}$ \\
\hline Constraint & $\left|t_{L}\right|=\left|t_{R}\right|$ & $r_{L}=r_{R}$ & $\left|r_{L}\right|=\left|r_{R}\right|$ & $\left|t_{L}\right|=\left|t_{R}\right|$ & $\left|t_{L}\right|=\left|t_{R}\right|,\left|r_{L}\right|=\left|r_{R}\right|$ & $\left|t_{L}\right|=\left|t_{R}\right|, r_{L}=r_{R}$ & None \\
\hline
\end{tabular}

These conclusions are straightforwardly applicable to explain the many intriguing symmetric/asymmetric scattering phenomena reported in the literatures. For example, the $C_{\mathbf{1}}$ symmetry protected symmetric transmission [2, 127]; the $C_{\mathcal{I}}$ symmetry protected symmetric reflection 7 , 8, 110, 128, 129, 140, 141; the $K_{1}$ symmetry protected symmetric reflection 44, 45]; the $K_{\mathcal{I}}$ symmetry protected symmetric transmission [45 95]; the $Q_{1}$ symmetry protected symmetric transmission and reflection [19, 130]; and the $P_{\mathcal{I}}$ symmetry protected symmetric transmission and reflection [36 43]. Without the protection of these symmetries, both the transmission and reflection are asymmetric [8, 9, 153].

\section{B: Details for the light scattering in the three-coupled-resonator scattering center}

The scattering center of the three coupled resonators is schematically illustrated in the main text Fig. 2(a). The scattering center encloses a synthetic magnetic flux $\phi$ induced by the Peierls phase factor in the coupling. We now analyze the properties of the scattering center through the two-port scattering dynamics. The leads 1 and 3 are under consideration; the lead 2 is disconnected from the scattering center.

For the case $\left\{V_{1}, V_{2}, V_{3}\right\}=\{i \gamma,-i \gamma, 0\}$, the Hamiltonian $H_{c}$ is not symmetry-protected. The scattering coefficients are obtained in the form of

$$
\begin{aligned}
& t_{L}=\frac{J\left(e^{2 i k}-1\right)\left(J e^{i \phi}+i \gamma+2 J \cos k\right)}{\left(2-e^{-2 i k}+e^{2 i k}+e^{i(k-\phi)}+e^{i(k+\phi)}\right) J^{2}+i J \gamma e^{i k}-\gamma^{2}}, \\
& r_{L}=-\frac{\left(1+e^{2 i k}+e^{i(k-\phi)}+e^{i(k+\phi)}\right) J^{2}+i J \gamma e^{-i k}-\gamma^{2}}{\left(2-e^{-2 i k}+e^{2 i k}+e^{i(k-\phi)}+e^{i(k+\phi)}\right) J^{2}+i J \gamma e^{i k}-\gamma^{2}}, \\
& t_{R}=\frac{J e^{-i \phi}\left(e^{2 i k}-1\right)\left(J+i \gamma e^{i \phi}+2 J e^{i \phi} \cos k\right)}{\left(2-e^{-2 i k}+e^{2 i k}+e^{i(k-\phi)}+e^{i(k+\phi)}\right) J^{2}+i J \gamma e^{i k}-\gamma^{2}}, \\
& r_{R}=-\frac{\left(1+e^{2 i k}+e^{i(k-\phi)}+e^{i(k+\phi)}\right) J^{2}+i J \gamma e^{3 i k}-\gamma^{2} e^{2 i k}}{\left(2-e^{-2 i k}+e^{2 i k}+e^{i(k-\phi)}+e^{i(k+\phi)}\right) J^{2}+i J \gamma e^{i k}-\gamma^{2}}
\end{aligned}
$$

The scattering coefficients are depicted in Figs. 2(b) and 2(c) of the main text; and they diverge at $J=\gamma / 2$ and $\phi= \pm \pi / 2$ for the resonant inputs at the momentum $k=-\pi / 2$. In the general case, the transmission and reflection are asymmetric; these are reflected from the four elements of the inverse matrix $\Delta^{-1}$

$$
\left(\begin{array}{cc}
\Delta_{m m}^{-1} & \Delta_{m n}^{-1} \\
\Delta_{n m}^{-1} & \Delta_{n n}^{-1}
\end{array}\right)=\frac{J^{2}}{\operatorname{det} \Delta}\left(\begin{array}{cc}
1+e^{2 i k}+e^{-2 i k}+i(\gamma / J)\left(e^{i k}+e^{-i k}\right) & e^{i k}+e^{-i k}+e^{-i \phi}+i(\gamma / J) \\
e^{i k}+e^{-i k}+e^{i \phi}+i(\gamma / J) & 1+e^{2 i k}+e^{-2 i k}+(\gamma / J)^{2}
\end{array}\right)
$$


where $\Delta=H_{c}-(2 J \cos k) \mathbf{1}$. The determinant is $\operatorname{det} \Delta=J^{3}\left[e^{-i \phi}+e^{i \phi}-e^{-3 i k}-e^{3 i k}-\left(\gamma^{2} / J^{2}\right)\left(e^{-i k}+e^{i k}\right)\right]$. Then, we obtain the contrast between the transmission and reflection of the inputs from the forward and backward directions for the resonant incidence with the momentum $k=-\pi / 2$

$$
\frac{t_{R}}{t_{L}}=\frac{e^{-i \phi}+i \gamma / J}{e^{i \phi}+i \gamma / J}, \frac{r_{R}}{r_{L}}=\frac{2 \cos \phi-i \gamma / J+i \gamma^{2} / J^{2}}{2 \cos \phi-i \gamma / J-i \gamma^{2} / J^{2}} .
$$

At $\gamma=J$, the special cases of $\phi=-\pi / 2$ for a unidirectional transmissionless $t_{L}=0, r_{L}=1 ; t_{R}=-2 i, r_{R}=0$ and $\phi=\pi / 2$ for a unidirectional absorption $t_{L}=-2 i, r_{L}=1 ; t_{R}=0, r_{R}=0$ are shown in the main text Fig. 3 .

\section{C: Non-Hermitian three-coupled-resonator scattering center with dissipative coupling}

In the coupled mode theory, the equations of motion for the three-site scattering center with dissipative coupling $-i \kappa$ are given by

$$
\begin{aligned}
& \dot{\phi}_{c}^{k}(1)=\omega_{0} \phi_{c}^{k}(1)-i \kappa \phi_{c}^{k}(2)+J \phi_{c}^{k}(3)+J \phi_{1}^{k}(-1), \\
& i \dot{\phi}_{c}^{k}(2)=\omega_{0} \phi_{c}^{k}(2)-i \kappa \phi_{c}^{k}(1)+J e^{-i \phi} \phi_{c}^{k}(3), \\
& i \dot{\phi}_{c}^{k}(3)=\omega_{0} \phi_{c}^{k}(3)+J \phi_{c}^{k}(1)+J e^{i \phi} \phi_{c}^{k}(2)+J \phi_{3}^{k}(1),
\end{aligned}
$$

where $\phi_{1}^{k}(-1)$ and $\phi_{3}^{k}(1)$ are the wavefunctions of the sites on the leads 1 and 3 that are connected with the scattering center sites 1 and 3, respectively. The dissipative coupling is reciprocal and is directly induced by the dissipation in the link resonator between the primary resonators 1 and 2 [114]. The link resonator and the primary resonators are on resonant. The effective dissipative coupling strength $\kappa=\kappa_{0}^{2} / \gamma$ is inversely proportional to the link resonator dissipation $\gamma$ and quadratically proportional to the coupling strength between the link resonator and the primary resonators $\kappa_{0}$ [113, 145]. The dissipative coupling has also been experimentally realized in many anti-parity-time symmetric systems [115, 142 144].

The scattering center is described by the Hamiltonian

$$
H_{c}=\left(\begin{array}{ccc}
0 & -i \kappa & J \\
-i \kappa & 0 & J e^{-i \phi} \\
J & J e^{i \phi} & 0
\end{array}\right)
$$

the scattering properties of which are not symmetry-protected.

The contrast between the transmission and reflection from opposite incident directions are calculated as follows. We obtain the four elements of the inverse matrix $\Delta^{-1}$ in the form of

$$
\left(\begin{array}{cc}
\Delta_{m m}^{-1} & \Delta_{m n}^{-1} \\
\Delta_{n m}^{-1} & \Delta_{n n}^{-1}
\end{array}\right)=\frac{J^{2}}{\operatorname{det} \Delta}\left(\begin{array}{cc}
e^{2 i k}+1+e^{-2 i k} & e^{i k}+e^{-i k}-i(\kappa / J) e^{-i \phi} \\
e^{i k}+e^{-i k}-i(\kappa / J) e^{i \phi} & e^{2 i k}+2+e^{-2 i k}+(\kappa / J)^{2}
\end{array}\right) .
$$

In general case, the transmission and reflection are asymmetric.

However, for the resonant incidence with the momentum $k=-\pi / 2$, the transmission is symmetric $\left|t_{L}\right|=\left|t_{R}\right|$ because of $\left|\Delta_{n m}^{-1}\right|=\left|\Delta_{m n}^{-1}\right|$; but the reflection is asymmetric. To break the reciprocity at $k=-\pi / 2$, the on-site terms $\left\{V_{1}, V_{2}, V_{3}\right\}$ of the scattering center are helpful

$$
H_{c}=\left(\begin{array}{ccc}
V_{1} & -i \kappa & J \\
-i \kappa & V_{2} & J e^{-i \phi} \\
J & J e^{i \phi} & V_{3}
\end{array}\right)
$$

For the resonant incidence $k=-\pi / 2$, the four elements of the inverse matrix $\Delta^{-1}$ are in the form of

$$
\left(\begin{array}{cc}
\Delta_{m m}^{-1} & \Delta_{m n}^{-1} \\
\Delta_{n m}^{-1} & \Delta_{n n}^{-1}
\end{array}\right)=\frac{1}{\operatorname{det} \Delta}\left(\begin{array}{cc}
V_{2} V_{3}-J^{2} & -J V_{2}-i J \kappa e^{-i \phi} \\
-J V_{2}-i J \kappa e^{i \phi} & V_{1} V_{2}+\kappa^{2}
\end{array}\right),
$$

where the determinant $D \equiv \operatorname{det} \Delta=V_{1} V_{2} V_{3}-J^{2} V_{1}-J^{2} V_{2}+\kappa^{2} V_{3}-i J^{2} \kappa\left(e^{-i \phi}+e^{i \phi}\right)$. 
At $\phi= \pm \pi / 2$, the transmission is asymmetric for the real $V_{2}$; the transmission is symmetric for the imaginary $V_{2}$. For the real $V_{1,2,3}$, the reflection is symmetric; otherwise, the reflection is asymmetric.

We take $V_{1}=V_{3}=0$ as an illustration. The scattering coefficients for the momentum $k=-\pi / 2$ are obtained in the form of

$$
\begin{aligned}
& t_{L}=\frac{J\left(e^{2 i k}-1\right)\left(-i V_{2}+\kappa e^{i \phi}+2 i J \cos k\right)}{i J^{2} e^{-2 i k}\left(e^{4 i k}+e^{2 i k}-1\right)+J\left(i V_{2} e^{-i k}-i V_{2} e^{i k}+\kappa e^{i(k-\phi)}+\kappa e^{i(k+\phi)}\right)-i \kappa^{2}}, \\
& r_{L}=-\frac{e^{-i \phi}\left(e^{i(k+\phi)} J-i \kappa\right)\left(i J e^{i k}+\kappa e^{i \phi}\right)}{i J^{2} e^{-2 i k}\left(e^{4 i k}+e^{2 i k}-1\right)+J\left(i V_{2} e^{-i k}-i V_{2} e^{i k}+\kappa e^{i(k-\phi)}+\kappa e^{i(k+\phi)}\right)-i \kappa^{2}}, \\
& t_{R}=\frac{i J e^{-i \phi}\left(e^{2 i k}-1\right)\left(-V_{2} e^{i \phi}-i \kappa+2 J e^{i \phi} \cos k\right)}{i J^{2} e^{-2 i k}\left(e^{4 i k}+e^{2 i k}-1\right)+J\left(i V_{2} e^{-i k}-i V_{2} e^{i k}+\kappa e^{i(k-\phi)}+\kappa e^{i(k+\phi)}\right)-i \kappa^{2}}, \\
& r_{R}=-\frac{e^{-i \phi}\left(e^{i(k+\phi)} \kappa+i J\right)\left(J e^{i \phi}-i \kappa e^{i k}\right)}{i J^{2} e^{-2 i k}\left(e^{4 i k}+e^{2 i k}-1\right)+J\left(i V_{2} e^{-i k}-i V_{2} e^{i k}+\kappa e^{i(k-\phi)}+\kappa e^{i(k+\phi)}\right)-i \kappa^{2}} .
\end{aligned}
$$

Both the scattering coefficients $t$ and $r$ diverge at $\phi= \pm \arccos \left(J^{2}-\kappa^{2}\right)(2 J \kappa)^{-1}$ and $V_{2}=0$ when the resonant momentum $k=-\pi / 2$. For the real $V_{2}=J$, we have the scattering coefficients $t_{L}=-2 i, r_{L}=-i ; t_{R}=0, r_{R}=i$ at $\phi=-\pi / 2$; and we have the scattering coefficients $t_{L}=0, r_{L}=-i ; t_{R}=-2 i, r_{R}=i$ at $\phi=\pi / 2$. The numerical simulations are shown in Fig. 4.
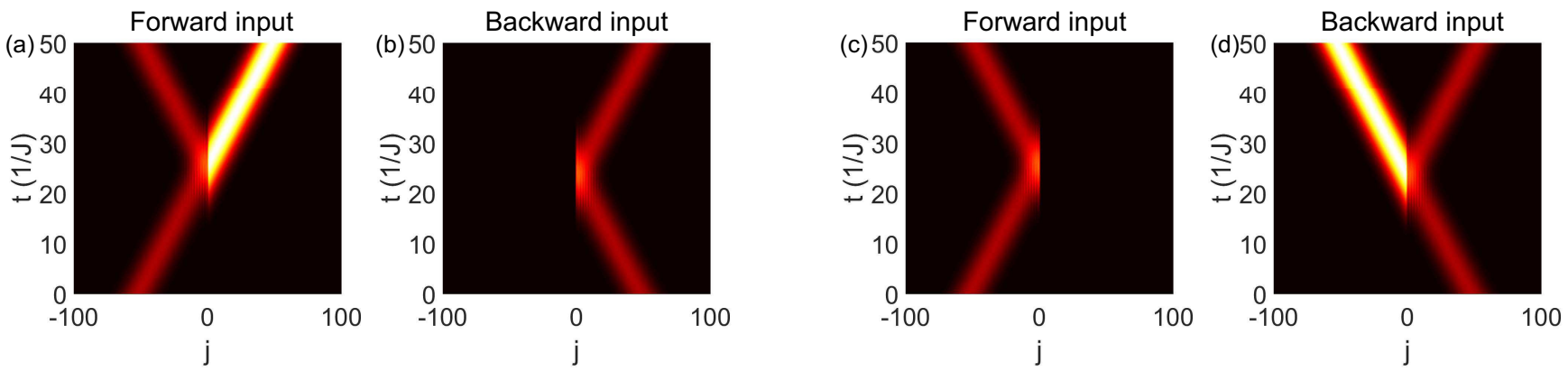

FIG. 4. Numerical simulation of the asymmetric transmission in the three-site center with dissipative coupling [Eq. (62)]. The incidences are Gaussian wave packet with the momentum $k=-\pi / 2$ in the simulations. The excitation intensity is depicted. (a) Forward and (b) backward incidence for $\phi=-\pi / 2 .\left|t_{L}\right|^{2}=4,\left|r_{L}\right|^{2}=1 ;\left|t_{R}\right|^{2}=0,\left|r_{R}\right|^{2}=1$. (c) Forward and (d) backward incidence for $\phi=\pi / 2$. $\left|t_{L}\right|^{2}=0,\left|r_{L}\right|^{2}=1 ;\left|t_{R}\right|^{2}=4,\left|r_{R}\right|^{2}=1$. The dissipative coupling is $\kappa=J=1$ and the on-site terms are $\left\{V_{1}, V_{2}, V_{3}\right\}=\{0, J, 0\}$. The incident frequency is $\omega_{0}$. 